\title{
Contractions of Angles in Symmetric Cones
}

\author{
To Jacques Faraut on his sixtieth birthday
}

By

Khalid KoufanY*

\begin{abstract}
For a symmetric cone $\Omega$ we compute its Riemannian distance in terms of the singular values of a generalized cross-ratio and prove that the semigroup of the compressions of $\Omega$ decreases the compounds distance.
\end{abstract}

\section{$\S 1 . \quad$ Introduction}

Let $S p(m, \mathbb{R})$ be the real symplectic group acting on the Siegel upper half plane $T_{\Omega_{S y m}}$ of complex symmetric matrices with positive definite imaginary part via the rational transformations

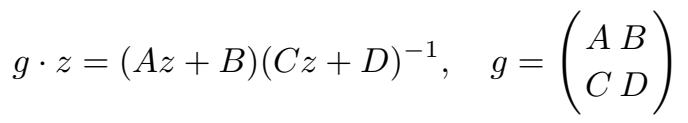

Let $\Omega_{\text {Sym }}$ be the set of real positive definite symmetric matrices. We can view it as the Riemannian symmetric space $G L(m, \mathbb{R}) / O(m)$. C. L. Siegel's study of the symplectic geometry [16] allows one to get an explicit formula for the geodesic distance in $\Omega_{S y m}$ associated with the $G L(m, \mathbb{R})$ invariant Riemannian metric, which coincides with the Euclidean arc length on the logarithms of

Communicated by M. Kashiwara. Received April 23, 2001.

2001 Mathematics Subject Classification(s): 17C50, 20M30, 32M15.

Key words: angles, contractions, cross-ratio, symmetric cones.

Author partially supported by the European Commission (TMR 1998-2001 Network Harmonic Analysis).

*Institut E. Cartan, Universié H. Poincaré, B.P. 239, F-54506 Vandœuvre-lès-Nancy.

e-mail: koufany@iecn.u-nancy.fr 
the diagonal matrices in $\Omega_{\text {Sym }}$. Namely, if $x, y \in \Omega_{\text {Sym }}$, then the Riemannian distance of $x$ and $y$ is given by

$$
\delta(x, y)=\left(\sum_{k=1}^{m} \log ^{2} \lambda_{k}(x, y)\right)^{1 / 2},
$$

where $\lambda_{1}(x, y) \geq \cdots \geq \lambda_{m}(x, y)>0$ are the characteristic value of $(x, y)$, i.e. the eigenvalues of the matrix $y^{-1} x$. The eigenvalues $\lambda_{k}(x, y)$ are the unique solutions of the equation

$$
\operatorname{Det}(x-\lambda y)=0 .
$$

Furthermore, if we put

$$
q(x, y):=(x-y)(x+y)^{-1}(x-y)(x+y)^{-1}
$$

then, the real numbers

$$
\xi_{j}(x, y):=\left(\frac{1-\lambda_{j}(x, y)}{1+\lambda_{j}(x, y)}\right)^{2}
$$

are the unique eigenvalues of the cross-ratio $q(x, y)$ and the formula (1.1) can be written as follows:

$$
\delta(x, y)=\left(\sum_{k=1}^{m} \log ^{2} \frac{1+\sqrt{\xi_{k}(x, y)}}{1-\sqrt{\xi_{k}(x, y)}}\right)^{1 / 2} .
$$

Let us consider now the set $\mathcal{H}$ of the matrices in $S p(m, \mathbb{R})$ that compress $\Omega_{\text {Sym }}$ :

$$
\mathcal{H}:=\left\{g \in S p(m, \mathbb{R}) \mid g \cdot \Omega_{\text {Sym }} \subset \Omega_{\text {Sym }}\right\} .
$$

It is a semigroup of $S p(m, \mathbb{R})$ and it contains $G L(m, \mathbb{R})$ and the inversion $x \mapsto x^{-1}$. One can show that it is the semigroup of the Hamiltonian matrices:

$$
\mathcal{H}=\left\{\left(\begin{array}{ll}
A & B \\
C & D
\end{array}\right) \mid A \text { invertible, } B A^{*} \geq 0, A^{*} C \geq 0\right\}
$$

where $M \geq 0$ means that $M$ is a positive symmetric matrix, i.e. $M \in \bar{\Omega}_{\text {Sym }}$. A natural question arises now : what is the behaviour of the angles $\mu_{k}(x, y):=$ $\log ^{2} \lambda_{k}(x, y)$ and therefore of the Riemannian distance upon the action of the semigroup $\mathcal{H}$ ? 
This problem was solved by $\mathrm{Ph}$. Bougerol in [1] where he proved that the elements of $\mathcal{H}$ are contractions (and sometimes uniform contractions) of the $\mu_{k}(x, y)$ and then for the Riemannian distance.

This problem has a good interpretation in Euclidean Jordan algebras and causal symmetric spaces theories. Indeed $\Omega_{S y m}$ is the symmetric cone associated with the Euclidean Jordan algebra $\operatorname{Sym}(m, \mathbb{R})$ and $\mathcal{H}$ is the causal semigroup associated with the ordered symmetric space $\operatorname{Sym}(m, \mathbb{R}) / G L(m, \mathbb{R}) \otimes \mathbb{R}$.

Let $V$ be a Euclidean Jordan algebra and $\Omega$ the symmetric cone associated with $V$. In this paper we shall deal with an explicit distance formula for $\Omega$. We call it Siegel's distance formula, since it has close analogies with formulas (1.1) and (1.5) introduced by C. L. Siegel for symmetric matrices. The Siegel distance formula involves a generalized cross-ratio for a quadruple

$$
D(w, x, y, z):=P(w-x) P(x-y)^{-1} P(z-y) P(z-w)^{-1}
$$

introduced for special cases by L. K. Hua and C. L. Siegel and generalized to any Jordan algebra by $\mathrm{H}$. Braun. The fundamental property of this cross-ratio is, as Siegel observed, that if $z$ and $w$ are two points of the tube $V+i \Omega$, then $D(z, w, \bar{z}, \bar{w})$ always has real eigenvalues.

The second aim of this paper is to study the monotone behaviour of the angles upon the action of the compression semigroup

$$
S=\{\gamma \in G \mid \gamma \cdot \Omega \subset \Omega\}
$$

where $G$ is the conformal group of $V$.

In Section 2 we collect basic facts about Euclidean Jordan algebras needed in this paper. In Section 3 we connect the characteristic values with the eigenvalues of a generalized cross-ratio. In Section 4 we give a generalization of a U. Hirzebruch theorem which is the Fischer min-max theorem on the eigenvalues of elements in $V$. In Section 5 we prove the Siegel distance formula for $\Omega$. In Section 6 we give some properties of the compression semigroup $S$ and finally in Section 7 we prove that the elements of the semigroup $S$ are contractions for the angles and then for the Riemannian distance, thus generalizing the result of Ph. Bougerol.

\section{$\S 2 . \quad$ Preliminaries}

Let $\Omega$ be an open convex cone in a Euclidean vector space $V$ of dimension $n$. Let $G(\Omega)$ be the group of linear automorphisms of $\Omega$

$$
G(\Omega)=\{g \in G L(V) \mid g \Omega=\Omega\} .
$$


Then $\Omega$ is said to be homogeneous if $G(\Omega)$ acts on it transitively. If $\bar{\Omega}$ is pointed, then $\Omega$ is said to be symmetric if it is homogeneous and self-dual.

A Euclidean Jordan algebra is a Euclidean vector space $V$ equipped with a bilinear product such that

$$
\begin{aligned}
x y & =y x, \\
x\left(x^{2} y\right) & =x^{2}(x y), \\
(x y \mid z) & =(y \mid x z) .
\end{aligned}
$$

It is shown in [11] and [17] that the interior $\Omega$ of the set of squares in $V$ is a symmetric cone, and every symmetric cone is given in this way.

We define the (left) multiplication $L$ by $L(x) y=x y$ and the so-called quadratic representation $P$ by $P(x)=2 L^{2}(x)-L\left(x^{2}\right)$. For any $x \in V$, the endomorphisms $L(x)$ and $P(x)$ are self-adjoint.

For example, $V=\operatorname{Sym}(m, \mathbb{R})$ is a Jordan algebra for the product $x \circ y=$ $(1 / 2)(x y+y x)$ and it is Euclidean for the scalar product $(x \mid y)=\operatorname{Tr}(x y)$. The symmetric cone is the cone $\Omega_{S y m}$ of positive definite symmetric matrices and $G\left(\Omega_{\text {Sym }}\right)$ is the linear group $G L(m, \mathbb{R})$. In this case, $P(x) y=x y x$.

For any $x \in \Omega, P(x)$ is positive definite and then the bilinear forms

$$
\mathrm{g}_{x}(u, v)=\left(P(x)^{-1} u \mid v\right), \quad x \in \Omega, \quad u, v \in V
$$

define on $\Omega$ a structure of Riemannian symmetric space isomorphic to $G(\Omega)_{\circ} /$ $K(\Omega)$ 。 where $G(\Omega)$ 。 is the identity component of $G(\Omega), K(\Omega) \circ=\{g \in G(\Omega) \circ \mid$ $g e=e\}$ and $e$ the identity element of $V$.

Let $r$ be the rank of $V$. A Jordan frame $\left\{c_{1}, \ldots, c_{r}\right\}$ of $V$ is a complete system of non-zero orthogonal primitive idempotents:

$$
\begin{aligned}
& c_{i}^{2}=c_{i}, \quad c_{i} \text { indecomposable, } \\
& c_{i} c_{j}=0 \quad \text { if } i \neq j, \\
& c_{1}+\cdots+c_{r}=e .
\end{aligned}
$$

Suppose $V$ is simple. In other words, there is no non-trivial ideal in $V$. Then each element $x$ in $V$ can be written as

$$
x=k \sum_{j=1}^{r} \lambda_{j} c_{j}, \quad k \in K(\Omega)_{\circ}, \quad \lambda_{j} \in \mathbb{R} .
$$

The determinant is defined by $\operatorname{det}(x)=\prod_{j=1}^{r} \lambda_{j}$ and the trace form by $\operatorname{tr}(x)=$ $\sum_{j=1}^{r} \lambda_{j}$. The real numbers $\lambda_{1}, \ldots, \lambda_{r}$ are the eigenvalues of $x$. 
In the case of $V=\operatorname{Sym}(m, \mathbb{R}),(2.1)$ corresponds to the polar decomposition (diagonalization) of symmetric matrices. det and tr are the usual determinant Det and trace $\mathrm{Tr}$ of matrices.

If $\lambda_{1}, \ldots, \lambda_{r}$ are the eigenvalues of $x$, then the eigenvalues of $P(x)$ are $\eta_{j k}:=\lambda_{j} \lambda_{k}$, where $j, k \in\{1, \ldots, r\}$. We will call the square roots of $\eta_{11}, \ldots$, $\eta_{r r}$ the singular values of $P(x)$. They are the absolute values of the eigenvalues of $x$.

Let $T_{\Omega}$ be the tube domain

$$
T_{\Omega}=V+i \Omega=\{z=x+i y \mid x \in V, y \in \Omega\} .
$$

It is a Hermitian symmetric space isomorphic to $G / K$ where $G$ is the group of holomorphic automorphisms of $T_{\Omega}$ and $K$ is the stabilizer of $i e$ in $G$.

In the case of $V=\operatorname{Sym}(m, \mathbb{R})$, the tube domain is the Siegel upper half plane $T_{\Omega_{S y m}}$, the group $G$ is the symplectic group $S p(m, \mathbb{R})$ and $K$ is the unitary group $U(m)$.

Let $c$ be the Cayley transform $c: z \mapsto(e-z)(e+z)^{-1}$. Then it is shown in [9], that $G / c G(\Omega)_{\circ} c$ is an ordered symmetric space of Cayley type and any symmetric space of Cayley type is obtained in this way (see [6] for the definition). One proves (see [10]) that the corresponding causal semigroup is the semigroup of compressions of $\Omega$ :

$$
S=\{\gamma \in G \mid g \cdot \Omega \subset \Omega\}
$$

and it has the following decomposition where $S=S^{+} G(\Omega)_{\circ} S^{-}, S^{+}$and $S^{-}$are Abelian semigroups which are isomorphic to the cone $\bar{\Omega}$. Using this decomposition and infinitesimal arguments we also proved in [10] that the elements of $S$ are contractions of the $G(\Omega)$-invariant Riemannian metric of $\Omega$.

\section{$\S 3 . \quad$ The Characteristic Values and Cross-ratio}

Let $x, y \in V$. A root of the characteristic equation

$$
\operatorname{det}(x-\lambda y)=0
$$

is called a characteristic value of the pair $(x, y)$.

Let $w, x, y, z \in V$, and let $D(w, x, y, z)$ be the generalized cross-ratio studied by H. Braun [2, p. 26] given by

$$
D(w, x, y, z):=P(w-x) P(x-y)^{-1} P(y-z) P(z-w)^{-1}
$$


when the appropriate inverses exist. This was first introduced by L. K. Hua [8, p. 452] for the symmetric, skew-symmetric and Hermitian matrices. See also Siegel [16, p. 3] and Maaß [13, p. 39] for symmetric matrices.

For $x, y \in \Omega$ we put

$$
\begin{aligned}
\rho(x, y) & =D(x, y,-x,-y), \\
& =P(x-y) P(x+y)^{-1} P(x-y) P(x+y)^{-1} .
\end{aligned}
$$

Observe that $\rho(x, y)$ is well defined, since $x+y$ is invertible because $x+y \in \Omega$.

Proposition 3.1. 1. For $x \in V$ and $y \in \Omega$, the characteristic equation $\operatorname{det}(x-\lambda y)=0$ always has $r$ real characteristic values $\lambda_{1}(x, y) \geq \cdots \geq \lambda_{r}(x, y)$.

2. For $x, y \in \Omega$, the cross-ratio $\rho(x, y)$ always has real eigenvalues and its $r$ singular values $\eta_{1}(x, y), \ldots, \eta_{r}(x, y)$ may be related to the characteristic values by

$$
\eta_{k}(x, y)=\left(\frac{1-\lambda_{k}(x, y)}{1+\lambda_{k}(x, y)}\right)^{2}
$$

Proof.

1. By the Spectral Theorem ([4, Theorem III.1.2]) there exist $g \in G(\Omega)$, a Jordan frame $\left\{c_{1}, \ldots, c_{r}\right\}$ and $r$ real numbers $\lambda_{1}(x, y) \geq, \ldots, \geq \lambda_{r}(x, y)$ such that $y=g \cdot e$ and $x=g \cdot \sum_{j=1}^{r} \lambda_{j}(x, y) c_{j}$. Then

$$
\begin{aligned}
\operatorname{det}(x-\lambda y) & =\operatorname{det}\left(g \cdot\left(\sum_{j=1}^{r} \lambda_{j}(x, y) c_{j}-\lambda e\right)\right) \\
& =\operatorname{Det}(g)^{\frac{r}{n}} \prod_{j=1}^{r}\left(\lambda_{j}(x, y)-\lambda\right) .
\end{aligned}
$$

This proves that $\lambda_{1}(x, y), \ldots, \lambda_{r}(x, y)$ are the unique solutions of the characteristic equation since $\operatorname{Det}(g) \neq 0$. Moreover, these solutions are exactly the eigenvalues of $P\left(y^{-1 / 2}\right) x$.

2. Using the fundamental formula

$$
P(P(y) x)=P(y) P(x) P(y)
$$

([4, Proposition II.3.3]) we obtain

$$
\begin{aligned}
\rho(x, y) & =P(x-y) P(x+y)^{-1} P(x-y) P(x+y)^{-1} \\
& =P\left(y^{\frac{1}{2}}\right) P(z-e) P(z+e)^{-1} P(z-e) P(z+e)^{-1} P\left(y^{-\frac{1}{2}}\right)
\end{aligned}
$$


where $z=P\left(y^{-1 / 2}\right) x$. But if $u$ and $v$ are in the associative sub-algebra $\mathbb{R}[z]$, then $P(u) P(v)=P(u v)$ ([4, the proof of Proposition II.2.2]). Thus

$$
\rho(x, y)=P\left(y^{\frac{1}{2}}\right) P\left((z-e)^{2}(z+e)^{-2}\right) P\left(y^{-\frac{1}{2}}\right) .
$$

Since $\lambda_{1}(x, y), \ldots, \lambda_{r}(x, y)$ are the unique eigenvalues of $z$, it follows from the last formula, that $\left(\left(1-\lambda_{j}(x, y)\right) /\left(1+\lambda_{j}(x, y)\right)\right)^{2}\left(\left(1-\lambda_{k}(x, y)\right) /\left(1+\lambda_{k}\right.\right.$ $(x, y)))^{2},(j, k) \in\{1, \ldots, r\}^{2}$, are the unique eigenvalues of the cross-ratio $\rho(x, y)$. Hence $\left(\left(1-\lambda_{j}(x, y)\right) /\left(1+\lambda_{j}(x, y)\right)\right)^{2}, j \in\{1, \ldots, r\}$ are the singular values of $\rho(x, y)$.

Example 3.1. If $V=\operatorname{Sym}(m, \mathbb{R})$, we saw that the quadratic representation is given by $P(x) y=x y x$. Then

$$
\begin{aligned}
\rho(x, y) w & =P(x-y) P(x+y)^{-1} P(x-y) P(x+y)^{-1} w \\
& =q(x, y) w q(x, y)^{t}
\end{aligned}
$$

where $q(x, y)$ is the cross-ratio

$$
q(x, y)=(x-y)(x+y)^{-1}(x-y)(x+y)^{-1}
$$

introduced in (1.3). Therefore the eigenvalues of $q(x, y)$ are the singular values $\xi_{1}(x, y), \ldots, \xi_{m}(x, y)$ of $\rho(x, y)$ (see $\left.(1.4)\right)$.

\section{§4. A Generalized Min-Max Theorem}

Let $V$ be a simple Euclidean Jordan algebra of rank $r \geq 2$. We consider the scalar product on $V$

$$
(x \mid y)=\operatorname{tr}(x y)
$$

and denote by $\mathcal{J}(V)$ the set of primitive idempotents of $V$. An element $c$ of $V$ is said to be almost primitive idempotent, if $c \neq 0$ and

$$
c^{2}=(e \mid c) c
$$

Let $\mathcal{J}^{a}(V)$ be the set of the almost primitive idempotents of $V$. Observe that

$$
\begin{aligned}
\mathcal{J}(V) & =\left\{c \in \mathcal{J}^{a}(V) \mid \operatorname{tr}(c)=1\right\} \\
& =\left\{\frac{c}{(e \mid c)} \mid c \in \mathcal{J}^{a}(V)\right\}
\end{aligned}
$$


and that for a fixed $x \in V$, the maximum (resp. minimum) of the function $(x \mid c)$ over $\mathcal{J}(V)$ coincides with the maximum (resp. minimum) of the Rayleigh quotient $(x \mid c) /(e \mid c)$ over $\mathcal{J}^{a}(V)$.

We state, in a different way, the following theorem due to U. Hirzerbruch, see $[7]$.

Theorem 4.1. For any $x$ in $V$, the eigenvalues $\lambda_{1}(x) \geq \lambda_{2}(x) \geq \cdots \geq$ $\lambda_{r}(x)$ of $x$, may be obtained as follows:

$$
\lambda_{1}(x)=\max _{c \in \mathcal{J}^{a}(V)} \frac{(x \mid c)}{(e \mid c)}, \quad \lambda_{r}(x)=\min _{c \in \mathcal{J}^{a}(V)} \frac{(x \mid c)}{(e \mid c)},
$$

and for $2 \leq k \leq r-1$,

$$
\lambda_{k}(x)=\min _{d_{1}, \ldots, d_{k-1} \in \mathcal{J}^{a}(V)} \max _{c \in V^{a}\left(d_{1}, \ldots, d_{k-1} ; 0\right)} \frac{(x \mid c)}{(e \mid c)},
$$

where $V^{a}\left(d_{1}, \ldots, d_{k-1} ; 0\right)=\left\{c \in \mathcal{J}^{a}(V) \mid c d_{1}=\cdots=c d_{k-1}=0\right\}$.

To prove a generalization of this theorem, we need the following

Lemma 4.1. 1. $\mathcal{J}^{a}(V)=\{c \in \bar{\Omega} \cup-\bar{\Omega} \mid \operatorname{rank}(c)=1\}$.

2. $G(\Omega)$ acts transitively on $\mathcal{J}^{a}(V)$.

3. Let $c, d \in \mathcal{J}^{a}(V)$. Then $(c \mid d)=0$ if, and only if, $c d=0$.

Proof. The assertions 1 and 2 follow from [4, Proposition IV.3.1].

The assertion 3 is a direct consequence of the following fact: If $x, y \in \bar{\Omega}$, then

$$
(x \mid y)=0 \Longleftrightarrow x y=0
$$

see [5, Satz 4.1].

Theorem 4.2. If $x \in V$ and $y \in \Omega$, then the characteristic values $\lambda_{1}(x, y) \geq \cdots \geq \lambda_{r}(x, y)$ of $(x, y)$ are given by:

$$
\lambda_{1}(x, y)=\max _{c \in \mathcal{J}^{a}(V)} \frac{(x \mid c)}{(y \mid c)}, \quad \lambda_{r}(x, y)=\min _{c \in \mathcal{J}^{a}(V)} \frac{(x \mid c)}{(y \mid c)}
$$

and for $2 \leq k \leq r-1$,

$$
\lambda_{k}(x, y)=\min _{d_{1}, \ldots, d_{k-1} \in \mathcal{J}^{a}(V)} \max _{c \in V^{a}\left(d_{1}, \ldots, d_{k-1} ; 0\right)} \frac{(x \mid c)}{(y \mid c)} .
$$


Proof. There exists $g \in G(\Omega)$ such that $y=g \cdot e$ and $x=g \cdot \sum_{j=1}^{r} \lambda_{j}(x$, y) $c_{j}$ for some Jordan frame $\left\{c_{1}, \ldots, c_{r}\right\}$. Then

$$
\begin{aligned}
\max _{c \in \mathcal{J}^{a}(V)} \frac{(x \mid c)}{(y \mid c)} & =\max _{c \in \mathcal{J}^{a}(V)} \frac{\left(g \cdot \sum_{i=1}^{r} \lambda_{i}(x, y) c_{i} \mid c\right)}{(g \cdot e \mid c)}, \\
& =\max _{c \in \mathcal{J}^{a}(V)} \frac{\left(\sum_{i=1}^{r} \lambda_{i}(x, y) c_{i} \mid g^{t} \cdot c\right)}{\left(e \mid g^{t} \cdot c\right)} .
\end{aligned}
$$

Now, from Lemma 4.1 it follows that $g^{t} \cdot \mathcal{J}^{a}(V)=\mathcal{J}^{a}(V)$. Hence

$$
\begin{aligned}
\max _{c \in \mathcal{J}^{a}(V)} \frac{(x \mid c)}{(y \mid c)} & =\max _{d \in \mathcal{J}^{a}(V)} \frac{\left(\sum_{i=1}^{r} \lambda_{i}(x, y) c_{i} \mid d\right)}{(e \mid d)}, \\
& =\lambda_{1}(x, y), \quad \text { by Theorem 4.1. }
\end{aligned}
$$

We use the same proof to get the extremal property of $\lambda_{r}(x, y)$.

Let $2 \leq k \leq r-1$ and define the quantity

$$
\alpha_{k}(x, y)=\min _{d_{1}, \ldots, d_{k-1} \in \mathcal{J}^{a}(V)} \max _{c d_{1}=\cdots=c d_{k-1}=0} \frac{(x \mid c)}{(y \mid c)} .
$$

Then

$$
\alpha_{k}(x, y)=\min _{d_{1}, \ldots, d_{k-1} \in \mathcal{J}^{a}(V)} \max _{c d_{1}=\cdots=c d_{k-1}=0} \frac{\left(\sum_{j=1}^{n} \lambda_{j}(x, y) c_{j} \mid g^{t} \cdot c\right)}{\left(e \mid g^{t} \cdot c\right)} .
$$

Setting $c^{1}=g^{t} c$ and $d_{j}^{1}=g^{-1} d_{j}$ this becomes using Lemma 4.1

$$
\begin{aligned}
\alpha_{k}(x, y) & =\min _{d_{1}^{1}, \ldots, d_{k-1}^{1} \in \mathcal{J}^{a}(V)} \max _{c^{1} d_{1}^{1}=\cdots=c^{1} d_{k-1}^{1}=0} \frac{\left(\sum_{j=1}^{n} \lambda_{j}(x, y) c_{j} \mid c^{1}\right)}{\left(e \mid c^{1}\right)}, \\
& =\lambda_{k}(x, y), \quad \text { by Theorem 4.1. }
\end{aligned}
$$

The following corollary generalizes the monotone property by U. Hirzebruch [7].

Corollary 4.1. Let $x, y \in \Omega$ and $v \in \bar{\Omega}$. For any $k \in\{1, \ldots, r\}$ we have

$$
\begin{aligned}
& \lambda_{k}(x+v, y) \geq \lambda_{k}(x, y), \\
& \lambda_{k}(x, y+v) \leq \lambda_{k}(x, y) .
\end{aligned}
$$

The inequalities (4.1) and (4.2) become strict when $v \in \Omega$. 


\section{$\S 5$. The Riemannian Distance of $\Omega$}

The family of bilinear forms $\mathrm{g}_{x}$ given by,

$$
\mathrm{g}_{x}(u, v)=\left(P(x)^{-1} u \mid v\right), \quad x \in \Omega, \quad u, v \in V,
$$

defines a $G(\Omega)$-invariant Riemannian metric on $\Omega$, see [4, Theorem III.5.3]. Therefore, $\Omega$ is a Riemannian symmetric space isomorphic to $G(\Omega)_{\circ} / K(\Omega)_{\circ}$.

Theorem 5.1. Let $x, y \in \Omega$. Then there exists a unique curve of shortest length joining $x$ and $y$. The length of this curve is given by

$$
\delta(x, y)=\left(\sum_{k=1}^{r} \log ^{2} \lambda_{k}(x, y)\right)^{1 / 2},
$$

where $\lambda_{1}(x, y), \ldots, \lambda_{r}(x, y)$ are the characteristic values of $(x, y)$.

$\delta(x, y)$ is the Riemannian distance of $x$ and $y$.

Proof. Following the proof of Theorem 4.2 we may assume without loss of generality that $x=\sum_{i=1}^{r} \lambda_{i}(x, y) c_{i}$ and $y=e$.

The unique curve of shortest length joining $e$ and $x$ is $\gamma(t)=\exp (t \log x)$ where $\log x=\sum_{i=1}^{r} \log \lambda_{i}(x, y) c_{i}$. Thus the Riemannian distance between $x$ and $e$ is

$$
\begin{aligned}
\delta(x, e) & =\int_{0}^{1}\left\{\mathrm{~g}_{\gamma(t)}(\dot{\gamma}(t), \dot{\gamma}(t))\right\}^{\frac{1}{2}} d t \\
& =\int_{0}^{1}\left(P\left(\gamma(t)^{-\frac{1}{2}}\right) \dot{\gamma}(t) \mid P\left(\gamma(t)^{-\frac{1}{2}}\right) \dot{\gamma}(t)\right)^{\frac{1}{2}} d t \\
& =\int_{0}^{1}\left(P\left(\gamma(t)^{-\frac{1}{2}}\right)\{\gamma(t) \log x\} \mid P\left(\gamma(t)^{-\frac{1}{2}}\right)\{\gamma(t) \log x\}\right)^{\frac{1}{2}} d t .
\end{aligned}
$$

Since $\log x, \gamma(t)$ and $\gamma(t)^{-1 / 2}$ are in the associative sub-algebra $\oplus_{k=1}^{r} \mathbb{R} c_{k}$, this implies that

$$
\begin{aligned}
\delta(x, e) & =\int_{0}^{1}\left(\left\{P\left(\gamma(t)^{-\frac{1}{2}}\right) \gamma(t)\right\} \log x \mid\left\{P\left(\gamma(t)^{-\frac{1}{2}}\right) \gamma(t)\right\} \log x\right)^{\frac{1}{2}} d t \\
& =\int_{0}^{1}(\log x \mid \log x)^{\frac{1}{2}} d t \\
& =\int_{0}^{1}\left(\sum_{k=1}^{r} \log \lambda_{k}(x, y) c_{k} \mid \sum_{k=1}^{r} \log \lambda_{k}(x, y) c_{k}\right)^{\frac{1}{2}} d t \\
& =\left(\sum_{k=1}^{r} \log ^{2} \lambda_{k}(x, y)\right)^{\frac{1}{2}} .
\end{aligned}
$$


Remark. $\delta$ is the Riemannian distance on $\Omega$ associated with the involution $x \mapsto x^{-1}$. The main property of this distance is its invariance by $G(\Omega)$.

For the symmetric cone of positive definite symmetric matrices and for the Siegel upper half plane this distance formula was obtained by C. L. Siegel [16, Theorem 3], see also Maaß [13, pp. 27, 39] and Neretin [15, Thoerem 6.3.5].

Corollary 5.1. Let $x, y \in \Omega$. Then the Riemannian distance of $x$ and y may be given by

$$
\delta(x, y)=\left(\sum_{k=1}^{r} \log ^{2} \frac{1+\sqrt{\eta_{k}(x, y)}}{1-\sqrt{\eta_{k}(x, y)}}\right)^{1 / 2},
$$

where $\eta_{1}(x, y), \ldots, \eta_{r}(x, y)$ are the singular values of the cross-ratio $\rho(x, y)$.

Proof. This is a direct consequence of Theorem 5.1 and Proposition 3.1.

\section{§6. Compression Semigroup}

Let $G$ be the conformal group of the Jordan algebra $V$. It is the group of all holomorphic automorphisms of the tube domain $T_{\Omega}=V+i \Omega$. The group $G$ is generated by the affine group $P$ of transformations

$$
z \mapsto g z+v \quad(g \in G(\Omega), v \in V)
$$

and by the symmetry,

$$
z \mapsto-z^{-1}
$$

The homogeneous space $G / P$ is compact, and $V$ can be embedded into $G / P$

$$
\begin{aligned}
V & \rightarrow G / P \\
v & \mapsto g P
\end{aligned}
$$

where $g(z)=-z^{-1}+v . G / P$ is the conformal compactification of $V$.

In the action of $G$ on $G / P$, we introduced (see [10]) the compression semigroup $S$ of $\Omega$ :

$$
S=\{\gamma \in G \mid \gamma \cdot \Omega \subset \Omega\}
$$

For $v \in \bar{\Omega}$, the translation

$$
\gamma_{v}^{+}(z)=z+v
$$


is a holomorphic automorphism of $T_{\Omega}$ and the semigroup of all real translations $\gamma_{v}^{+}$is an Abelian sub-semigroup $S^{+}$of $S$ isomorphic to $\bar{\Omega}$.

Put $j: z \mapsto z^{-1}$. This is an isometry of $\Omega$. Let $S^{-}=j \circ S^{+} \circ j$, be the semigroup of the maps

$$
\gamma_{v}^{-}(z)=\left(z^{-1}+v\right)^{-1}, \quad v \in \bar{\Omega} .
$$

It is an Abelian sub-semigroup of $S$ isomorphic to $\bar{\Omega}$.

Theorem 6.1 ([10]). $\quad$ The sub-semigroups $S^{+}$and $S^{-}$, together with the subgroup $G(\Omega)_{\circ}$, generate $S$. More precisely, one has the following decomposition

$$
S=S^{+} G(\Omega)_{\circ} S^{-} .
$$

If $\gamma=\gamma_{u}^{+} g \gamma_{v}^{-} \in S$, then we write

$$
n^{+}(\gamma):=u, \quad A(\gamma):=g \quad \text { and } \quad n^{-}(\gamma):=v
$$

Remark. We have also the other side decomposition

$$
S=S^{-} G(\Omega)_{\circ} S^{+}
$$

\section{$\S 7 . \quad$ Contraction Semigroup}

It is shown in [10], by using infinitesimal arguments, that the elements of $S$ are contractions of the $G(\Omega)$-invariant Riemannian metric (5.1) on $\Omega$.

The aim of this section is to study the monotone behaviour of the angles:

$$
\mu_{k}(x, y):=\log ^{2} \lambda_{k}(x, y)
$$

and prove refined contraction properties of the Riemannian distance (5.2) of $\Omega$.

Recall that for $x \in V$, the number $\sup \{(x \mid c) \mid c \in \mathcal{J}(V)\}$, which is the largest eigenvalue of $x$ (see Theorem 4.1), defines the (operator) norm $|x|$ of $x$.

Lemma 7.1. Let $x, y \in V$.

1. If $x-y \in \Omega$, then $|x| \geq|y|$.

2. If $x, y \in \Omega$, then $x-y \in \Omega$ if, and only if, $y^{-1}-x^{-1} \in \Omega$.

Proof. Observe that every element in $\Omega$ is invertible. 
1. If $x-y \in \Omega$, then for any $c \in \mathcal{J}(V),(x-y \mid c)>0$ and thus $|x| \geq|y|$.

2. Let $x, y \in \Omega$, then there exist $g \in G(\Omega)$, a Jordan frame $\left\{c_{1}, \ldots, c_{r}\right\}$ and $r$ real numbers $\lambda_{j}>0$ such that $x=g^{t} \cdot e$ and $y=g^{t} \cdot \sum_{j=1}^{r} \lambda_{j} c_{j}$. Since $x-y \in \Omega$ we have $1>\lambda_{j}$. Furthermore,

$$
\begin{aligned}
y^{-1}-x^{-1} & =g^{-1} \cdot \sum_{j=1}^{r} \frac{1}{\lambda_{j}} c_{j}-g^{-1} \cdot e, \\
& =g^{-1} \cdot\left(\sum_{j=1}^{r}\left(\frac{1}{\lambda_{j}}-1\right) c_{j}\right) \in \Omega .
\end{aligned}
$$

Conversely, if $y^{-1}-x^{-1} \in \Omega$, then $x-y \in \Omega$.

If $V=\mathbb{R}$ then $\Omega=\mathbb{R}_{+}^{*}$ and, for any $x, y \in \Omega$, the equation $x-\lambda y=0$ has only one solution $\lambda=x / y$ (recall $r=1$ in this case). Consider the distance between $x$ and $y$ given by $\delta(x, y)=|\log (x / y)|=|\log x-\log y|$. Let $r \geq 0$ (i.e. $r \in \bar{\Omega})$ and compare $\lambda(x+r, y+r)$ to $\lambda(x, y)$. By Rolle's theorem, there exists $c$, between $x$ and $y$, such that

$$
\frac{\log (x+r)-\log (y+r)}{\log x-\log y}=\frac{c}{c+r} .
$$

Therefore, if $0<x, y \leq m$,

$$
\log \frac{x+r}{y+r} \leq \frac{m}{m+r} \log ^{+} \frac{x}{y}
$$

where $\log ^{+} s=\max \{\log s, 0\}$. So that,

$$
\log ^{2} \lambda(x+r, y+r) \leq\left(\frac{m}{m+r}\right)^{2} \log ^{2} \lambda(x, y),
$$

and

$$
\delta(x+r, y+r) \leq \frac{m}{m+r} \delta(x, y) .
$$

This example will be used to prove following fundamental proposition:

Proposition 7.1. Let $x, y \in \Omega$ and $v \in \bar{\Omega}$. Then for any $k \in\{1, \ldots, r\}$,

$$
\left|\log \lambda_{k}(x+v, y+v)\right| \leq \frac{\alpha}{\alpha+\beta}\left|\log \lambda_{k}(x, y)\right|,
$$

where $\alpha=\max \{|x|,|y|\}$ and $\beta=\inf \{(v \mid c), c \in \mathcal{J}(V)\}$. 
Proof. In proving the inequality (7.3) we may assume $\lambda_{k}(x+v, y+v)>1$. Indeed, $\lambda_{k}(x+v, y+v)=1 / \lambda_{r-k+1}(y+v, x+v)$, moreover (7.3) is obvious when $\lambda_{k}(x+v, y+v)=1$.

Let $\lambda_{k}(x+v, y+v)>1$. Then we have for any $2 \leq k \leq r-1$

$$
\begin{aligned}
0 & <\left|\log \lambda_{k}(x+v, y+v)\right| \\
& =\log \lambda_{k}(x+v, y+v) \\
& =\log \min \left\{\max \left\{\frac{(x+v \mid c)}{(y+v \mid c)} \cdots\right\}, \cdots\right\}, \text { by Theorem } 4.2 \\
& =\min \left\{\max \left\{\log \frac{(x \mid c)+(v \mid c)}{(y \mid c)+(v \mid c)} \cdots\right\}, \cdots\right\} \\
& \leq \frac{\alpha}{\alpha+\beta} \min \left\{\max \left\{\log ^{+} \frac{(x \mid c)}{(y \mid c)} \cdots\right\}, \cdots\right\}, \text { by }(7.2) \\
& =\frac{\alpha}{\alpha+\beta} \log \lambda_{k}(x, y) \\
& =\frac{\alpha}{\alpha+\beta}\left|\log \lambda_{k}(x, y)\right| .
\end{aligned}
$$

To prove the inequatily (7.3) for $\lambda_{1}$ and $\lambda_{r}$ we use the same arguments.

Remark. If $v \in \bar{\Omega}$, then $\beta=\inf \{(v \mid c), c \in \mathcal{J}(V)\}$ may be equal to zero, and

$$
\left|\log \lambda_{k}(x+v, y+v)\right| \leq\left|\log \lambda_{k}(x, y)\right| .
$$

If $v \in \Omega$, then $v$ is invertible and $\beta$, the lowest eigenvalue of $v$, does not vanish. Hence

$$
\left|\log \lambda_{k}(x+v, y+v)\right|<\left|\log \lambda_{k}(x, y)\right| .
$$

Using the notations (6.3), we set

$$
S_{1}=\left\{\gamma \in S \mid n^{+}(\gamma) \in \Omega\right\},
$$

and

$$
S_{2}=\left\{\gamma \in S \mid n^{-}(\gamma) \in \Omega\right\}
$$

We state now the main theorem of this section.

Theorem 7.1. Let $k \in\{1, \ldots, r\}$. The following holds:

1. For any $\gamma \in S$ and for any $x, y \in \Omega: \mu_{k}(\gamma \cdot x, \gamma \cdot y) \leq \mu_{k}(x, y)$.

2. For any $\gamma \in S_{1} \cup S_{2}$ and for any $x, y \in \Omega: \mu_{k}(\gamma \cdot x, \gamma \cdot y)<\mu_{k}(x, y)$. 
3. For any $\gamma \in S_{1} \cap S_{2}$, there exists $\kappa(\gamma), 0<\kappa(\gamma)<1$, such that for any $x, y \in \Omega: \mu_{k}(\gamma \cdot x, \gamma \cdot y) \leq \kappa(\gamma) \mu_{k}(x, y)$.

Proof. Let $\gamma \in S$, then by Theorem 6.1 ,

$$
\begin{aligned}
\gamma & =\gamma_{u}^{+} g \gamma_{v}^{-} \\
& =\gamma_{u}^{+} g j \gamma_{v}^{+} j
\end{aligned}
$$

Here $u, v \in \bar{\Omega}, g \in G(\Omega)$ o and $j$ is the involution $j(w)=w^{-1}(w \in \Omega)$.

Recall that the elements of $G(\Omega)$ 。 are isometries of $\mu_{k}$. Moreover, the Hua identity [4, Lemma X.4.4]:

$$
\operatorname{det}\left(z^{-1}-w^{-1}\right)=(\operatorname{det} z)^{-1}(\operatorname{det} w)^{-1} \operatorname{det}(w-z)
$$

allows one to get $\lambda_{k}\left(x^{-1}, y^{-1}\right)=1 / \lambda_{k}(x, y)$. Therefore, $j$ is also an isometry of $\mu_{k}$. Hence, according to Proposition 7.1 and Remark 7, the first point of the theorem is proved.

Now if $u$ or $v$ is in the cone $\Omega$, then by considering again Remark 7 , the second point of the theorem follows.

It remains to prove the last point of the theorem. Let $\gamma=\gamma_{u}^{+} g \gamma_{v}^{-}=$ $\gamma_{u}^{+} g j \gamma_{v}^{+} j \in S_{1} \cap S_{2}$, then $u, v \in \Omega$. Since $j$ is an isometry of $\mu_{k}$, we have

$$
\begin{aligned}
\mu_{k}(\gamma \cdot x, \gamma \cdot y) & =\mu_{k}\left(\left(\gamma_{u}^{+} g j \gamma_{v}^{+}\right) \cdot x,\left(\gamma_{u}^{+} g j \gamma_{v}^{+}\right) \cdot y\right), \\
& =\mu_{k}\left(\left(g j \gamma_{v}^{+}\right) \cdot x+u,\left(g j \gamma_{v}^{+}\right) \cdot y+u\right) .
\end{aligned}
$$

According to Lemma 7.1, we obtain

$$
g \cdot v^{-1}-\left(g j \gamma_{v}^{+}\right) \cdot x=g \cdot v^{-1}-g \cdot(v+x)^{-1} \in \Omega,
$$

and

$$
\left|\left(g j \gamma_{v}^{+}\right) \cdot x\right| \leq\left|g \cdot v^{-1}\right|, \text { for any } x \in \Omega .
$$

Let $\zeta=\left|g \cdot v^{-1}\right|, \eta=\inf \{(u \mid c) \mid c \in \mathcal{J}(V)\}, \quad$ and put $\kappa(\gamma)=(\zeta /(\zeta+\eta))^{2}$. Then, it follows from Proposition 7.1 that

$$
\begin{aligned}
\mu_{k}(\gamma \cdot x, \gamma \cdot y) & \leq \kappa(\gamma) \mu_{k}\left(\left(g j \gamma_{v}^{+}\right) \cdot x,\left(g j \gamma_{v}^{+}\right) \cdot y\right) \\
& \leq \kappa(\gamma) \mu_{k}(x, y) .
\end{aligned}
$$

So that the theorem is proved.

As an easy consequence, Theorem 7.1 implies that the elements of the semigroup $S$ are contractions of the distance $\delta$. More precisely we have 
Corollary 7.1. The following holds:

1. For any $\gamma \in S$, and $x, y \in \Omega: \delta(\gamma \cdot x, \gamma \cdot y) \leq \delta(x, y)$.

2. For any $\gamma \in S_{1} \cup S_{2}$ and $x, y \in \Omega: \delta(\gamma \cdot x, \gamma \cdot y)<\delta(x, y)$.

3. For any $\gamma \in S_{1} \cap S_{2}$, there exists $\kappa(\gamma)$, $0<\kappa(\gamma)<1$, such that, for all $x, y \in \Omega: \delta(\gamma \cdot x, \gamma \cdot y) \leq \kappa(\gamma) \delta(x, y)$.

\section{§8. Final Remarks}

We refer to $[6]$ for the following definitions. Let $\mathcal{M}=G / H$ be a compactly causal symmetric space. Then $\mathcal{D}=G / K$ is a Hermitian symmetric domain. We can view $H / H \cap K$ as the real points of $\mathcal{D}$, i.e. fixepoint set of a conjugation on $\mathcal{D}$. For example, when $\mathcal{M}$ is a causal symmetric space of Cayley type, then $H / H \cap K$ is isomorphic to a symmetric cone $\Omega$ and $\mathcal{D}$ isomorphic to the tube domain $V+i \Omega$.

It is easy to see that the Siegel distance formula (5.3) extends to $V+i \Omega$, namely

$$
\delta(z, w)=\left(\sum_{j=1}^{r} \log ^{2} \frac{1+\sqrt{\eta_{j}(z, w)}}{1-\sqrt{\eta_{j}(z, w)}}\right)^{2}
$$

where $\eta_{1}(z, w), \ldots, \eta_{r}(z, w)$ are the singular values of the cross-ratio

$$
D(z, w, \bar{z}, \bar{w})=P(z-w) P(w-\bar{z})^{-1} P(\bar{z}-\bar{w}) P(\bar{w}-z)^{-1} .
$$

J.-L. Clerc proved that the elements of the Olshanskiu holomorphic semigroup $\Gamma=\left\{\gamma \in G_{\mathbb{C}} \mid \gamma \mathcal{D} \subset \mathcal{D}\right\}$ are contractions of $\mathcal{D}$ for the Bergman metric, see [3].

We conjecture that the Olshanskiu holomorphic semigroup decreases the angles

$$
\mu_{j}(z, w):=\log ^{2} \frac{1+\sqrt{\eta_{j}(z, w)}}{1-\sqrt{\eta_{j}(z, w)}} .
$$

This was proved, in the particular cases of $G=S p(n, \mathbb{R})$ and $G=U(p, q)$ by Neretin, see [14] and [15].

We also conjecture that the Siegel distance formula and the contractions properties, with respect to the associated compression semigroup, hold for the real bounded domain $H / H \cap K$.

Many applications of these results could be expected. For example to modular forms, filtering theory, control theory, Riccati equation theory, etc... 
After this paper was finished, the author noted that Y. Lim [12] proved independently that the elements of the semigroup $S$ are contractions of the largest angle $\mu_{1}$.

\section{Acknowledgement}

The author wishes to express his gratitude to J.-L. Clerc and B. Ørsted for useful remarks. He would like to thank the department of mathematics and computer science at Southern Denmark University-Odense, for the hospitality during his stay there in February 2000 when this work was being completed. $\mathrm{He}$ is also gratefull to the referee for many remarks.

\section{References}

[1] Bougerol, Ph., Kalman filtering with random coefficients and contractions, Geometric methods in representation theory, SIAM J. Control Optim., 32 (1993), 942-942.

[2] Braun, H., Doppelverhältnisse in Jordan-Algebren, Abh. Math. Sem. Univ. Hamburg, 32 (1968), 2-51.

[3] Clerc, J.-L., Compressions and contractions of Hermitian symmetric spaces, Math. Z., 229 (1998), 1-8.

[4] Faraut, J. and Korányi, A., Analysis on Symmetric Cones, Oxford Math. Monogr., Clarendon Press, Oxford, 1994.

[5] Hertneck, C., Positivitätsbereiche und Jordan-Strukturen, Math. Ann., 146 (1962), 433455.

[6] Hilgert, J. and Ólafsson, G., Causal symmetric spaces. Geometry and harmonic analysis, Perspect. Math., 18, Academic Press, Inc., San Diego, CA, 1997.

[7] Hirzebruch, U., Der Min-Max-Satz von E. Fischer fuer formal-reelle Jordan-Algebren, Math. Ann., 186 (1970), 65-69.

[8] Hua, L. K., Geometries of matrices. I. Generalizations of von Staudt's theorem, Trans. Amer. Math. Soc., 57 (1945), 441-481.

[9] Koufany, K., Réalisation des espaces symétriques de type Cayley, C. R. Acad. Sci. Paris Sér. I Math., 318 (1994), 425-428.

[10] - Semi-groupe de Lie associé à un cône symétrique, Ann. Inst. Fourier, 45 (1994), $1-29$.

[11] Koecher, M., Die Geodätischen von Positivitätsbereichen, Math. Ann., 135 (1958), 192202.

[12] Lim, Y., Finsler metric on symmetric cones, Math. Ann., 316 (2000), 379-389.

[13] Maaß, H., Siegel's Modular Forms and Dirichlet Series, Lecture Notes in Math., 216, Springer-Verlag, 1971.

[14] Neretin, Yu. A., On a semigroup of operators in the boson Fock space, Funct. Anal. Appl., 24 (1990), 135-144.

[15] - Categories of symmetries and infinite-dimensional groups, London Mathematical Society Monographs, Oxford Publications, Clarendon Press, 16 (1996).

[16] Siegel, C. L., Symplectic geometry, Amer. J. Math., 65 (1943), 1-86.

[17] Vinberg, E. B., Homogeneous cones, Soviet Math. Dokl., 1 (1960), 787-790. 